Open Access

\title{
The aerosol impact spectrometer: a versatile platform for studying the velocity dependence of nanoparticle-surface impact phenomena
}

\author{
Brian D. Adamson ${ }^{2}$, Morgan E. C. Miller ${ }^{1}$ and Robert E. Continetti2 ${ }^{2 *}$
}

\author{
${ }^{*}$ Correspondence: \\ rcontinetti@ucsd.edu \\ ${ }^{2}$ Department of Chemistry and \\ Biochemistry, University of \\ California, San Diego, 9500 Gilman \\ Drive, La Jolla, CA 92093-0340, USA \\ Full list of author information is \\ available at the end of the article
}

\begin{abstract}
A new apparatus designed to accelerate/decelerate and study the surface impact phenomena of charged aerosols and nanoparticles over a wide range of mass-tocharge $(\mathrm{m} / \mathrm{z})$ ratios and final velocities is described. A nanoparticle ion source coupled with a linear electrostatic trap configured as an image charge detection (ICD) mass spectrometer allows determination of the mass-to-charge ratio and the absolute charge and mass of single nanoparticles. A nine-stage linear accelerator/ decelerator is used to fix the final velocity of the nanoparticles, and in the results reported here the coefficient of restitution for polystyrene latex spheres (PSLs) impacting on silicon is measured using ICD techniques. To enable this apparatus to study a wide range of $\mathrm{m} / \mathrm{z}$, the data acquisition system uses a transient digitizer interfaced to a field-programmable gate array module that allows real time calculation of $\mathrm{m} / \mathrm{z}$ and determination of the pulse sequence for the linear accelerator/decelerator. Electrospray ionization of a colloidal suspension of PSL spheres of 510 and $990 \mathrm{~nm}$ has been used to demonstrate acceleration and deceleration of charged nanoparticles and the resolution of the apparatus. Measurements of the coefficient of restitution for PSLS on silicon over the range $10-400 \mathrm{~m} / \mathrm{s}$ are consistent with previous studies.
\end{abstract}

Keywords: Image charge detection, Nanoparticle acceleration, Deceleration, Coefficient of restitution

\section{Introduction}

As interest in the characterization of nanoparticles, aerosols and dusts increases, the need for the development of new tools for the manipulation and analysis of single particles continues to grow [1]. An area of continued interest for an understanding of problems ranging from atmospheric chemistry to astrophysical phenomena to industrial applications pertains to the impact phenomena of nanoparticles. Theoretical and experimental studies of nanoparticle-surface collisions at both low and high velocity ranges continue to be reported [2-7]. There is a large body of work on hypervelocity impact phenomena dating back to the early 1960's motivated by the need to understand the effect of cosmic dust and micrometeoroid impacts on space vehicles. Larger objects are most conveniently accelerated with light-gas guns [8], however, for smaller particles that can be easily charged, electrostatic accelerators are a convenient

(c) The Author(s); licensee Springer on behalf of EPJ. 2017 Open Access This article is distributed under the terms of the Creative Commons Attribution 4.0 International License (http://creativecommons.org/licenses/by/4.0/), which permits unrestricted use, distribution, and reproduction in any medium, provided you give appropriate credit to the original author(s) and the source, provide a link to the Creative Commons license, and indicate if changes were made. 
approach [9-12]. In early work in this area, a $2 \mathrm{MV}$ van de Graaff dust accelerator was described and shown to accelerate $1 \mu \mathrm{m}$ particles to $\sim 6 \mathrm{~km} / \mathrm{s}[10,11]$. One early example of a switched, multistage linear accelerator for large particles was described by Vedder [13], however it was not until advances in high-voltage switching circuitry that laboratory-scale linear accelerators, such as the one described by Hendell and Even [14], became more common. In the present work the development of a versatile new nanoparticle mass spectrometer/accelerator/decelerator, the Aerosol Impact Spectrometer (AIS), is described with a demonstration of its capabilities using polystyrene latex (PSL) spheres, including studies of collision inelasticity on silicon wafers by determination of incident and scattered velocities in measurements of the coefficient of restitution.

The key to carrying out mass spectrometric measurements on single charged nanoparticles is the use of charge detection mass spectrometry (CDMS) techniques (1). CDMS determines the absolute charge on a particle from the magnitude of the image charge induced on a pickup electrode when a charged particle passes through. The image charge waveform also yields the particle time-of-flight (TOF) and velocity through the pickup providing the mass-to-charge ratio for fixed energy particles [15]. This method has been used in accelerator experiments since at least 1960 [9], and with the increasing interest in studies of massive biomolecules, cells and nanoparticles it has seen more recent applications in mass spectrometry measurements [16-24], with the state of the art recently described by Keifer and Jarrold [25]. In our own laboratory, we make use of a variant of this approach, applying a charge-pickup electrode in an electrostatic fast-ion beam trap to monitor the ion density oscillating in the trap and carry out Fourier-transform (FT) mass spectrometry on ensembles of molecular ions [26, 27]. In the present apparatus the $\mathrm{m} / \mathrm{z}$ ratio of a single particle provides the information required to accelerate or decelerate that particle for studies of particle impact.

As shown by Hendell and Even [14], and later applied by Hsu and co-workers [28], a linear accelerator for large molecular ions can be configured using modern high-voltage MOSFET switching techniques. In the present apparatus, the CDMS determines the $m / z$ ratio for each particle in real time, providing the information required to accelerate or decelerate single particles over a wide range of $\mathrm{m} / \mathrm{z}$ by generating the appropriate acceleration/deceleration pulsed waveform for a linear accelerator (LINAC) structure composed of a series of cylindrical electrostatic elements. Acceleration of ion ensembles by this technique is not hindered by space charge limitations since the effect of space charge is progressively reduced as the particles accelerate. Deceleration of ion ensembles does become problematic as a result of increasing space charge, and that is the benefit of working with single charged nanoparticles where this limitation is not present.

In the following sections, the initial results obtained with the AIS will be presented, showing the measurement of charge/mass distributions, acceleration and deceleration of single charged PSL spheres, and measurements of impact inelasticity of single particles with a silicon substrate. These results show that the AIS will have great utility for measuring the reflection of nanoparticles from surfaces, yielding quantitative information on the coefficient of restitution that describes the inelasticity of such collisions $[29,30]$.

\section{Experimental apparatus}

A schematic of the AIS is shown in Fig. 1. An electrospray ionization (ESI) ion source and beam line including an aerodynamics lens (ADL) is orthogonal to the axis of the 


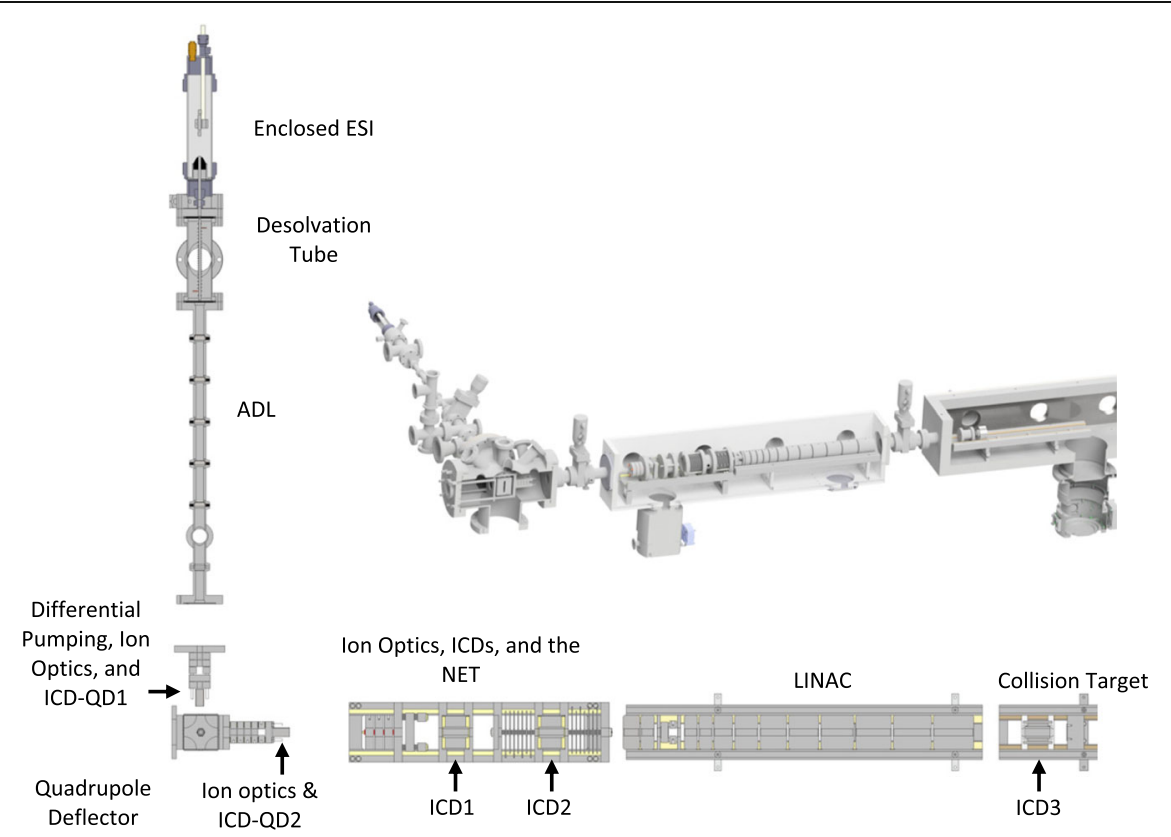

Fig. 1 Schematic and perspective view of the Aerosol Impact Spectrometer. Components starting from the charged aerosol source: Enclosed electrospray ionization (ESI); Desolvation tube; Aerodynamic Lens (ADL); Differential pumping, ion optics and image charge detector ICD-QD1; Quadrupole Deflector (QD), ion optics and ICD-QD2; Injection optics for Nanoparticle Electrostatic Trap (NET), including ICD1; NET, including ICD2; Injection optics; and 9-stage Linear Accelerator (LINAC); Final ICD (ICD3) and collision target. Locations of image charge detection tubes are indicated with a black arrow

nanoparticle electrostatic trap (NET) and the LINAC. A quadrupole deflector (QD) is used to provide an energy selected beam of single nanoparticles for analysis in the NET. The QD can be operated over a wide energy range, selecting different particle distributions as determined by the size, charge and ESI expansion conditions. Characterization of the NET, the LINAC, and the initial measurements of impact dynamics are detailed in the following paragraphs.

Charged particles are formed by electrospray ionization and transferred into a low vacuum through a $150-\mu \mathrm{m}$ Pt electron microscope aperture. A heater tube dries the particles before they enter the ADL, which collimates and focuses the particle beam. The particle beam passes through two stages of differential pumping and enters a chamber that houses the electrostatic quadrupole deflector. The QD selectively turns particles $90^{\circ}$ based on their kinetic energy per charge into the next chamber that contains the NET. The NET is a linear electrostatic trap configured as an image charge mass spectrometer, as first described by Zajfman and co-workers on ion ensembles [31, 32] and in a single-particle application by Benner [1]. Briefly, the NET is gated to trap one particle at a time, before measuring the mass-to-charge ratio and the absolute charge of the particle. These particles can be trapped in the NET with an efficiency $\sim 70 \%$ for periods in excess of 5 seconds with a distribution of oscillation frequencies in the several hundred Hertz range. A Labview-based data acquisition code uses the $m / z$ data to calculate the required switching times for the LINAC to accelerate the particle up to the required velocity. The particle is then released from the trap into the LINAC for acceleration to the desired velocity. 


\section{Electrospray ionization (ESI) source}

The use of ESI for this type of application is based on prior work by Benner, Austin and others on polystyrene latex spheres, silica, mineral dusts and other systems [3336]. The ESI source consists of a $360 \mu \mathrm{m}$ O.D., $75 \mu \mathrm{m}$ I.D. fused silica capillary mounted on an isolated aluminum block, which is held at approximately $+4-6 \mathrm{kV}$. To date the AIS instrument has only been run in positive mode, but no impediment is anticipated for using it to study negatively charged particles. The tip of the capillary is positioned $\sim 10 \mathrm{~mm}$ in front of a $150 \mu \mathrm{m}$ aperture and is enclosed within a glass tube. The aperture is mounted on a $6.4 \mathrm{~mm}$ OD, $3.86 \mathrm{~mm}$ ID stainless steel tube that passes through an Ultra-Torr fitting into the instrument. The portion of this tube that extends out of the vacuum chamber is surrounded by an enclosure, through which nitrogen, heated to $\sim 85{ }^{\circ} \mathrm{C}$, flows. The heated nitrogen helps to desolvate the particles, and also acts as a curtain gas. $510 \mathrm{~nm}$ (Polyscience \#07307) and $990 \mathrm{~nm}$ (Polyscience \#07310) PSL spheres were suspended in 1:1 mixtures of $25 \mathrm{mM}$ aqueous ammonium acetate and methanol, then delivered to the electrospray tip by a syringe pump operating at a rate of $0.26 \mathrm{~mL} / \mathrm{h}$. The final number densities were $30 \times 10^{9}$ particles per $\mathrm{mL}$ for the $510 \mathrm{~nm}$ PSL suspension, and $4.1 \times 10^{8}$ particles per $\mathrm{mL}$ for the $990 \mathrm{~nm}$ suspension. After entering the vacuum chamber, the particles pass through a $120 \mathrm{~mm}$ long, $4.5 \mathrm{~mm}$ I.D. stainless steel tube, which is heated to $\sim 185{ }^{\circ} \mathrm{C}$ to completely desolvate the particles, and to evaporate charged solvent droplets.

\section{Aerodynamic lens (ADL)}

After exiting the heater tube, the particles enter an ADL, which aerodynamically focuses and collimates the beam of particles [37, 38]. In this instance the ADL consists of 5 apertures separated by $59 \mathrm{~mm}$ long, $12.1 \mathrm{~mm}$ I.D. spacers. The diameters of the apertures are 5.9, 5.3, 4.6, 3.4 and $3.1 \mathrm{~mm}$. The section after the $5^{\text {th }}$ aperture is connected to a mechanical vacuum pump. The pressure at the start of the ADL is 2.6 Torr, and the pressure in the pumped region is typically in the range of $0.5-1$ Torr. The precise pressure after the ADL is adjusted, by throttling the vacuum pump, in order to maximize transmission of particles in a specific size range. Calculations along the line of those described by Wang and McMurry[39] indicate that this ADL should transmit over $95 \%$ of particles in the range of $75-1200 \mathrm{~nm}$.

\section{Quadrupole deflector (QD)}

Following the ADL, the particles pass through a $3.1 \mathrm{~mm}$ diameter aperture into the first differential pumping stage, which is connected to a Roots blower backed by a rotary mechanical pump. This pumping stage also contains an image charge detector tube (ICD) to confirm transmission of particles through the aerodynamic lens. A $5 \mathrm{~mm}$ aperture leads to the second differential pumping stage, pumped by a Pfeiffer TMH 064 turbomolecular pump. A $3.2 \mathrm{~mm}$ aperture separates the second differential pumping stage from the quadrupole deflector chamber, which is pumped by an Osaka TG240 turbomolecular pump. The quadrupole deflector chamber is pumped to a vacuum of $3 \times 10^{-5}$ Torr.

The QD assembly is in the center of the chamber and consists of 4 parallel, quartercylinder stainless steel rods (19 mm radius). The rods are mounted on $1 / 8$ " precision 
ground glass spheres that locate the rods such that their curved faces are tangential to an inscribed circle of $33.7 \mathrm{~mm}$ diameter. Positive and negative potentials are applied to opposing pairs of rods, resulting in an electrostatic field that will turn particles that have kinetic energy, in $\mathrm{eV}$ per charge, equivalent to the potential applied to the rods. Particles with excess kinetic energy will overshoot the bend, whereas particles with less kinetic energy will impact the electrode on the inside of the curve.

There are two sets of ion optics in the chamber with the QD, each comprised of an einzel lens, an $x-y$ deflector and an ICD, modeled after the detector presented by Fuerstenau and Benner [16]. One is positioned before the entrance to the QD, the other after the exit from the QD. These ion optics serve to focus the selected particles and direct them to the next chamber, which contains the NET and LINAC. The first ICD (ICD-QD1) is used to confirm transmission of particles through the ADL. The second ICD (ICD-QD2) is used to determine the optimal deflector potential to direct the particle beam towards the next chamber.

\section{Nanoparticle electrostatic trap}

After the QD, the energy-selected particles pass into the NET chamber through another set of electrostatic optics (einzel lens and $x-y$ deflectors) mounted on an isolated rail system (Fig. 2). This assembly is mounted in a vacuum chamber that is pumped by another Osaka TG240 turbomolecular pump and achieves vacuum of $10^{-6}$ Torr. Another ICD is located directly after the optics stack to track transmission of particles into the NET chamber. The ICD connects directly to the junction field-effect transistor (JFET) input of an Amptek A250 charge-sensitive amplifier circuit mounted in vacuum on the grounding plates shielding the detector. Output from the ICD circuit passes to a feedthrough where it can be monitored externally.

After passing through this ICD tube, particles are transmitted into the NET. The design of the NET (Fig. 3) was modeled after the ion trap originally developed by Benner [1]. Two electrostatic mirrors each composed of four individual elements connected by a network of external potentiometers establish the fields that trap the charged particles. The mirror elements have an outer diameter of $63.5 \mathrm{~mm}$, a beam-line inner diameter of $9.7 \mathrm{~mm}$ and a thickness of $1.5 \mathrm{~mm}$. Each mirror stack incorporates an additional three

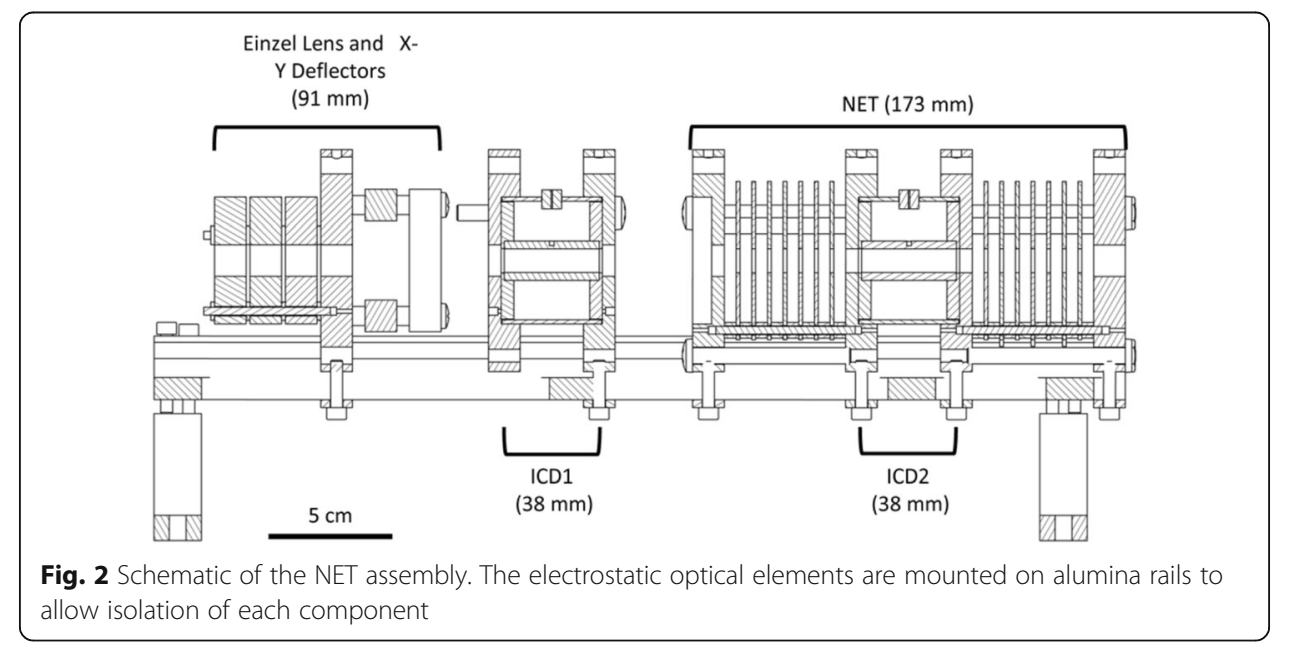




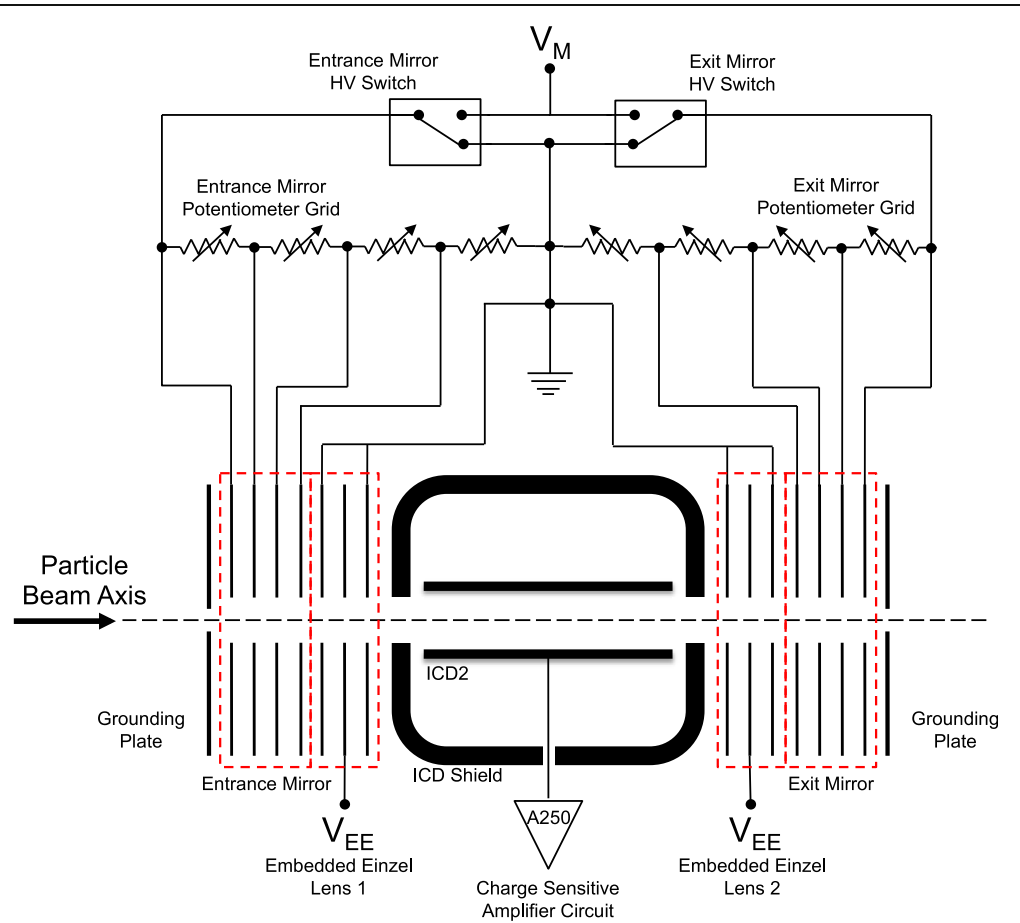

Fig. 3 Diagram of the NET. $V_{M}$ is the potential applied to the NET mirrors. $V_{E E}$ is the potential applied to the embedded einzel. Separate potentiometer networks with separate HV switches control the two mirror potentials independent of each other, allowing for complete control of trapping potentials

elements that act as an intracavity einzel lens to refocus trapped particles. The NET has an ICD (ICD2) mounted in the field-free region centered between the two, sevenelement mirror stacks. ICD2 is a $38.1 \mathrm{~mm}$ long tube with an outer diameter of $15.9 \mathrm{~mm}$ and an inner diameter of $9.5 \mathrm{~mm}$. The tube is isolated with PEEK insulators that mount on the final grounded einzel element of each mirror stack. The elements in each mirror stack are spaced by $4.8 \mathrm{~mm}$ nylon spacers mounted on alumina rods that hold the seven elements in place. The entire assembly is enclosed by two grounded plates and mounted on the isolated rail system with a total length of $173 \mathrm{~mm}$. Each mirror stack is wired to a multipin feedthrough that connects to an external network of potentiometers. Each network is set up as a multistep voltage divider that controls the potential and field shape of each mirror stack independent of the other. The potentiometer networks are wired to two home-built high voltage $(4 \mathrm{kV})$ MOSFET switches that control the mirror potentials. Potentials used on the NET mirrors are the same polarity as the charge of the particles being trapped. The intracavity-einzel lens can be operated in either polarity to establish a stable potential for trapping. SIMION 8.1.1 was used to generate a model of the field used in this experiment for the NET as shown in Fig. 4 [40]. ICD2 is wired directly to the junction field-effect transistor (JFET) input of an Amptek A250 charge sensitive amplifier circuit. This circuit is mounted in a shielded box directly above the assembly, and the output is connected with shielded coaxial cable to a coaxial feedthrough.

The signal from the NET ICD2 is connected to a home-built shaping amplifier for baseline subtraction and signal gain. The output of this amplifier is connected to an FPGA (National Instruments PXI-7952R) with a high-speed digitizer front end 


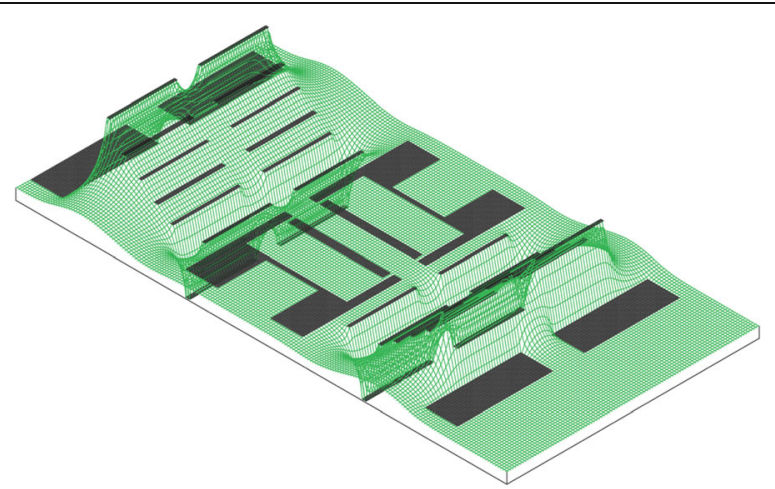

Fig. 4 SIMION (8.1) representation of the potentials in the NET. A positive potential is used on the outer NET mirrors to trap positive particles. Two einzel lenses operating with a negative focusing potential are mounted on either side of the central ICD tube to continually re-focus particles within the trap

(National Instruments NI 5731) and an oscilloscope (Tektronix DPO 3034) (Fig. 5). The overall gain of the A250 preamplifier and shaping amplifier is determined by applying a $5 \mathrm{mV}$ trapezoidal waveform, with rise and fall times similar to those of the waveforms induced by the oscillating particles, through a $1.5 \mathrm{pF}$ capacitor attached directly to the ICD pickup. The capacitor converts the known voltage to a known charge, and the amplified response to the known input provides the overall gain calibration factor. As currently configured this amplifier has successfully detected particles with as low as 1000 charges. Based on variation in the response to the calibration input signal, the 1 standard deviation uncertainty on the charge measurement is roughly 100 charges. The initial state of the trap has the entrance mirror potential lowered, and the exit mirror potential raised. When a particle passes through ICD2 in the trap, the output pulse from the charge-sensitive amplifier triggers closing of the entrance mirror. With both mirrors raised the charged particle oscillates in the trap producing a regular signal from ICD2 with a measurable amplitude and frequency. This signal is captured by the FPGA digitizer and analyzed by a LabView code while the particle is still

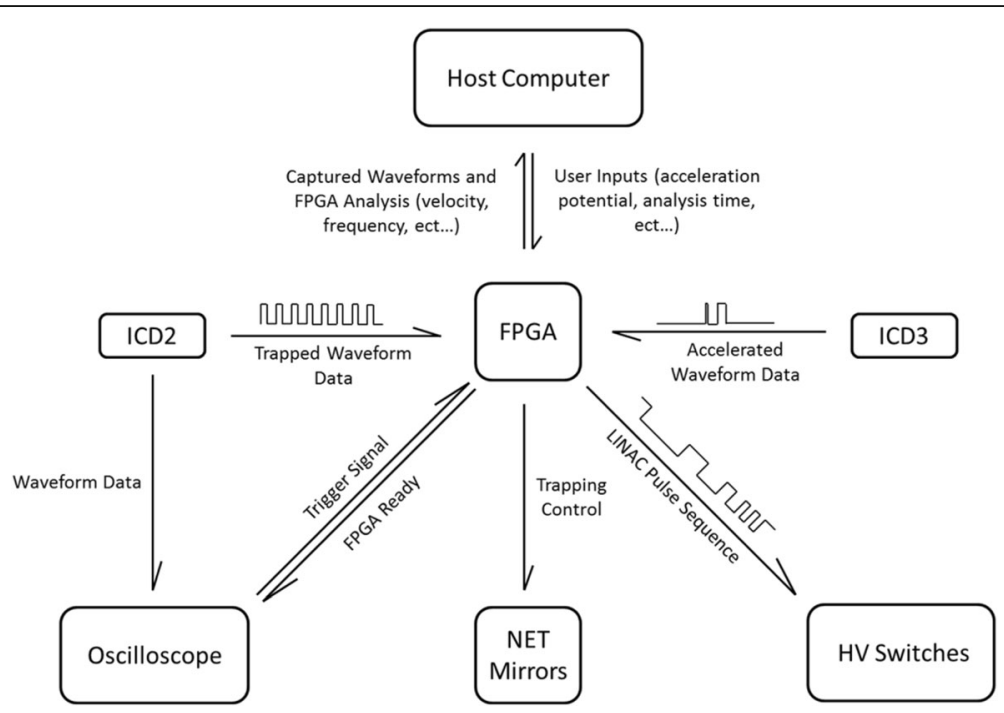

Fig. 5 Schematic of the Aerosol Impact Spectrometer data acquisition and computer control system 
trapped. The $m / z$ ratio of the particle in the trap is determined from its oscillation frequency, $f$, using the following relationship $[1,26]$ :

$$
m / z=\frac{C}{f^{2}}
$$

The calibration factor $C$ is dependent on the trapping potentials of the NET and the kinetic energy-per-charge of the trapped particle. This factor is calculated using a SIMION simulation with these parameters [40]. In addition to frequency, the FPGA calculates the velocity of the particle in the trap by measuring the temporal width of the output pulses $\left(t_{\text {pulse width }}\right)$ from ICD2 of length $L_{I C D 2}$ :

$$
v_{\text {particle }}=\frac{t_{\text {pulse width }}}{L_{I C D 2}}
$$

This data is measured and calculated on the fly for each particle and is used to create the timing sequence used by the HV switches for acceleration/deceleration.

\section{Linear accelerator}

Directly after the NET there is a pair of $\mathrm{x}-\mathrm{y}$ deflectors, mounted on a second isolated rail system in the same chamber. This rail system has the same layout and mounting as the NET, and allows the LINAC to be removed from the chamber independently of the NET. After the deflectors, twelve cylindrical polished stainless steel electrodes are mounted $1.7 \mathrm{~mm}$ apart on the remaining length of the rail. The accelerator electrodes all have a $50.8 \mathrm{~mm}$ outer diameter, a $10.16 \mathrm{~mm}$ inner diameter, and beveled edges. The first four electrodes are all the same length $\left(L_{1}=20.32 \mathrm{~mm}\right)$, with the length of each subsequent element determined by the following relationship:

$$
L_{n}=L_{1} * \sqrt{n} ; n=\{2,3,4, \ldots, 9\}
$$

The first three electrodes act as a lens with each element connected to external power supplies (two KIKUSUI PMC350-0.2A and one Canberra 3002). The remaining 9 electrodes are wired through two HV feedthroughs $(30 \mathrm{kV})$ with every second element collectively wired to one feedthrough and every other element collectively wired to the other feedthrough. All elements in each set are connected together with copper rods. These two rods are connected to each HV feedthrough with a shielded HV cable. The elements are pulsed with two $30 \mathrm{kV}$ HV switches (Behlke 301-03-GSM).

To calculate the timing used to trigger the two HV switches, the data acquisition program uses the $m / z$ of the particle and the initial velocity to perform a simulation of the particle travelling through the LINAC. This is accomplished be using a positiondependent electric field equation derived from a SIMION model of the LINAC electrodes. A code steps through time and velocity to calculate the acceleration of the particle under the influence of the electric field:

$$
t_{x}=t_{x-1}+\left(\frac{d x}{v_{x-1}}\right) \text { and } v_{x}=v_{x-1}+\left(\frac{E_{x-1}}{m / z} *\left(t_{x}-t_{x-1}\right)\right)
$$

In Eq (4), $t_{x}$ is the time at position $x, v_{x}$ is the velocity at position $x, E_{x}$ is the electric field at position $x$, and $d x$ is the step size of the calculation, in this case $0.01 \mathrm{~mm}$. This 
timing calculation is performed while the particle is still trapped in the NET. Upon completion of the calculation, timing data is transferred to the FPGA. The FPGA then releases the particle from the trap by lowering the exit mirror and triggers the two high voltage (HV) switches connected to the LINAC at the times required to accelerate/decelerate the particle to a final energy that depends on the number of elements used and the potential applied to each element.

\section{Collision target}

After the LINAC rail system, another shielded ICD tube (ICD3) is mounted on an isolated rail system of the same design as the NET and LINAC rails. This rail system is in a chamber pumped by an Osaka TG420 to a vacuum of $10^{-7}$ Torr. The ICD is placed $19.8 \mathrm{~mm}$ in front of an aluminum block on which a collision target is mounted. The collision target used in this experiment was a $2.5 \mathrm{~cm}$ square piece of p-type silicon (cut from a polished, 6 inch diameter, $600 \mu \mathrm{m}$ thick boron doped wafer, Ziti Inc.). An Amptek A250 charge sensitive amplifier circuit is located directly above the ICD tube, and the output is connected to the FPGA digitizer through a second home-built baseline subtraction and amplification circuit to capture signals from ICD3. The target and ICD3 are positioned such that particles rebounding at a maximum angle of $5.2^{\circ}$ from center can be measured by ICD3 for incident and rebounding beam axis velocity as shown in Fig. 6.

After acceleration, the FPGA digitizer captures a waveform from the output of ICD3. The waveform is transferred to the Labview program which calculates the accelerated velocity of the particle. The program also determines if the particle has rebounded from the collision target, and calculates the rebound velocity of the particle from the rebounding peak width. After completing this final acquisition the program saves all information to a data file and resets itself and the FPGA to accept a new particle. The entire run time for each particle in this experiment was approximately $\sim 200 \mathrm{~ms}$.

\section{Results and discussion}

\section{Charge distributions in 510 and 990 nm PSL colloids}

Electrospray ionization of PSLs produces particles that carry a wide range of elementary charges.[41-43] The QD selects a subset of these particles for injection into the

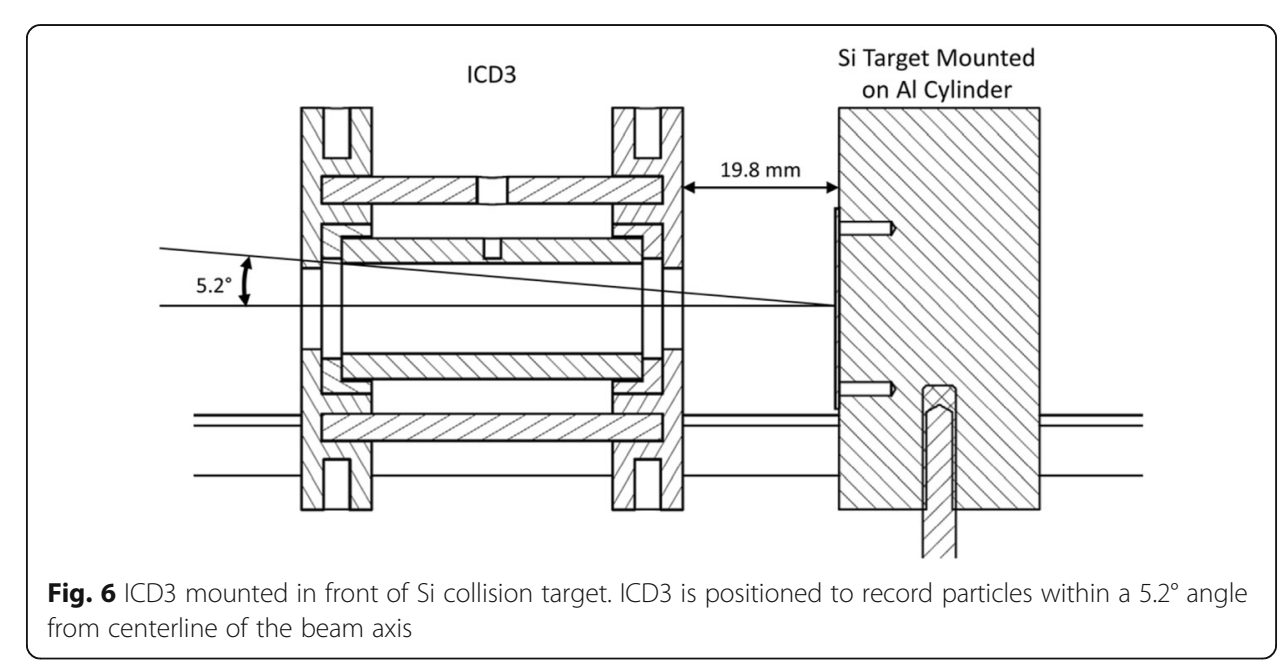


NET and measurement with ICD2. The Rayleigh limit [44] for a charged liquid governs the maximum charge a particle can acquire when generated by electrospray ionization [45]. To avoid Coulombic explosion, the total charge of a liquid sphere, $q$, must satisfy:

$$
q \leq 8 \pi \sqrt{\epsilon_{0} \gamma a^{3}}
$$

where $\epsilon_{0}$ is the permittivity of free space, $\gamma$ is the surface tension of the liquid, and $a$ is the radius of the liquid droplet. The surface tension can be approximated as the surface tension of a 50:50 $\mathrm{H}_{2} \mathrm{O}: \mathrm{CH}_{3} \mathrm{OH}$ solution. At the desolvation gas temperature of $\sim 85{ }^{\circ} \mathrm{C}$ used in these experiments the surface tension of this solvent is estimated to be $26.56 \mathrm{mN} / \mathrm{m}$ [46]. For the particle sizes presented here the Rayleigh limits in these conditions are 9,800 and 26,500 elementary charges, $z$, for the 510 and $990 \mathrm{~nm}$ PSLs, respectively.

Figure 7 shows a 2D colormap of the number of $990 \mathrm{~nm}$ PSL particles passing through the ICD-QD2, immediately after the QD, plotted against the QD potential and amplitude of the image charge signal for each particle. ICD-QD2 was not calibrated directly, but comparison to charge measurements performed on ICD2 shows that the Rayleigh limit of 26500 charges would correspond to a peak height of $0.7 \mathrm{~V}$ on ICDQD2. The region of interest is the curved band (the large horizontal feature correlated with lower peak heights corresponds to particles that have impacted the surface of ICD-QD2, rather than passing through). The inverse relationship between the amplitude of the image charge signal and QD potential is consistent with particles presenting with a constant total kinetic energy, which in turn is consistent with particles of similar size, travelling at similar velocities. At low QD potentials, particles with a large number of charges are selected, with the number of charges limited by the Rayleigh limit. At high QD potentials, the kinetic energy is partitioned among fewer charges, leading to the detection of correspondingly smaller amplitude image charge signals.

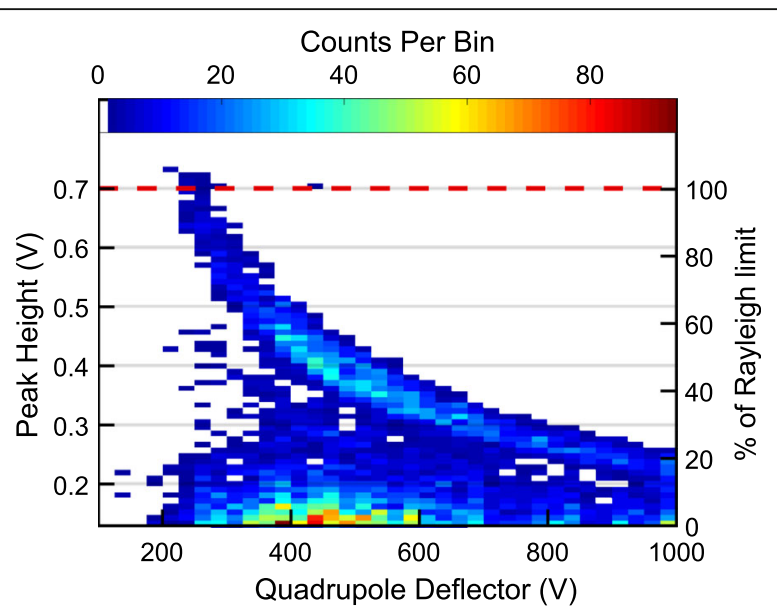

Fig. 72 histogram of peak height of $990 \mathrm{~nm}$ PSLs arriving at ICD-QD2, plotted against QD potential. ICD-QD2 is not directly calibrated, but the calibration factor can be inferred by reference to another detector (ICD2). The average number of charges on a $990 \mathrm{~nm}$ PSL as measured by ICD2 for particles selected with the QD set to $400 \mathrm{~V}$ is 17000 . This corresponds to a peak height of $0.45 \mathrm{~V}$ on ICD-QD2, so the Rayleigh limit of 26500 charges would correspond to about $0.7 \mathrm{~V}$ (as shown by the dashed red line). The large number of peaks with heights of $<0.2 \mathrm{~V}$ are due to particles that have hit the detector pickup instead of passing all the way through 
In Fig. 8 the measured charge distributions for $510 \mathrm{~nm}$ and $990 \mathrm{~nm}$ particles are shown. In these measurements, the QD was set to $70 \mathrm{~V}$ for selection of $510 \mathrm{~nm}$ particles, and to $400 \mathrm{~V}$ for $990 \mathrm{~nm}$ particles. The charge measurement is performed by calculating the mean peak height in volts of the image charge waveform induced by the trapped particle (Fig. 9) in a $64 \mathrm{~ms}$ data acquisition window. The amplitude of the acquired waveform is converted to a measure of charge using the gain calibration factor. These charge measurements fall below the Rayleigh limit for each species and above previous measurements reported for smaller PSLs [47].

Using Eq. (1), the measured charge and frequency can be used to calculate the mass of the trapped particles. For the PSL samples studied here, the expected mass-perparticle is in the Gigadalton range: $43.9 \mathrm{GDa}\left(7.3 \times 10^{-17} \mathrm{~kg}\right)$ for $510 \mathrm{~nm}$ PSLs, and 321.3 GDa (5.34 x $\left.10^{-16} \mathrm{~kg}\right)$ for $990 \mathrm{~nm}$ PSLs. These experimental values for the particle mass are in good agreement with the expected values, as shown in Fig. 10 for the particle charge distributions shown in Fig. 8.

\section{Variable acceleration and deceleration of charged nanoparticles}

The variable energy linear accelerator allows for a wide range of particle final velocities to be achieved. Particles are accelerated/decelerated to a final velocity $\left(v_{f}\right)$ according to the following relationship between their initial energy-per-charge $\left(E_{0}\right)$, LINAC electrode potential $\left(E_{L}\right)$, number of LINAC elements used $(n)$, and initial velocity $\left(v_{i}\right)$ :

$$
\frac{v_{f}}{v_{i}}=\sqrt{\frac{E_{0}+n E_{L}}{E_{0}}} .
$$

The initial energy is determined by the potential set on the QD, and the initial velocity is determined by the pressure differential across the ADL and the QD chamber. For accelerating voltages the LINAC electrode potential in the above equation is treated as a positive quantity which results in an increase in final velocity $\left(v_{f}>v_{i}\right)$. When decelerating particles the LINAC electrode potential is treated as a negative quantity. In the experiments presented here (using positively charged particles) acceleration was accomplished using negative potentials on the LINAC ranging from $0 \mathrm{~V}$ to

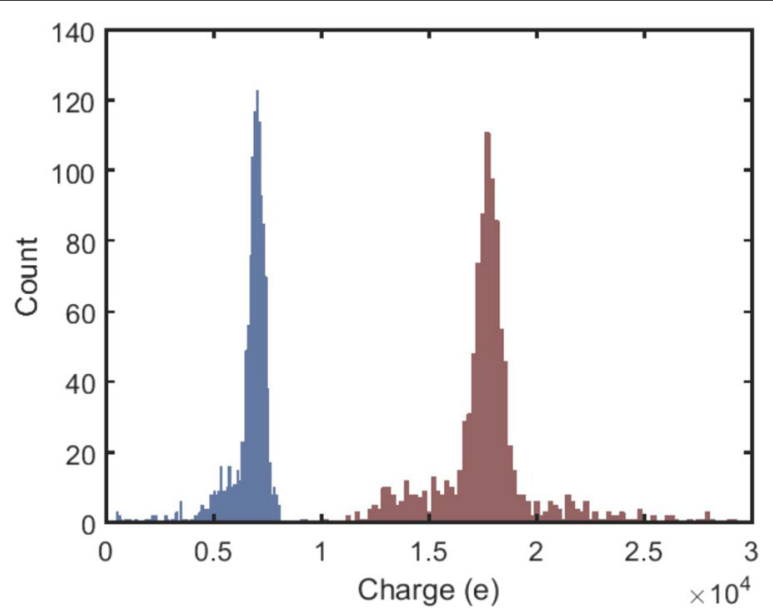

Fig. 8 Charge distribution of $510 \mathrm{~nm}$ PSL (blue) and $990 \mathrm{~nm}$ PSL (red) species, energy selected for $70 \mathrm{eV} / \mathrm{z}$ and $400 \mathrm{eV} / \mathrm{z}$, respectively 


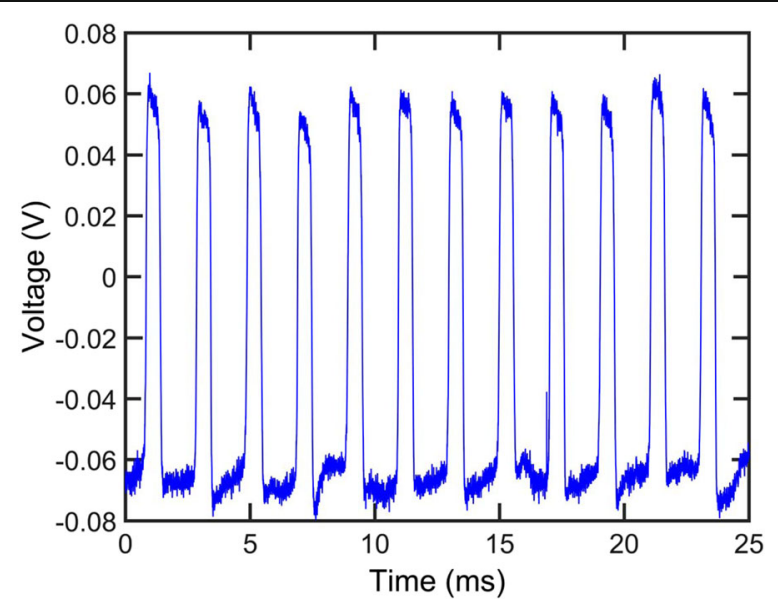

Fig. 9 Portion of typical ICD2 trace for a $990 \mathrm{~nm}$ PSL oscillating in the NET. Acquisition captures all peaks, overlays them and finds the mean peak height to calculate particle charge

$-45.0 \mathrm{kV}$ (nine stages at $-5 \mathrm{kV} /$ stage). Results using an acceleration potential $-22.5 \mathrm{kV}$ are shown in Fig. 11. Deceleration of particles was accomplished using positive potentials on the LINAC ranging from $0 \mathrm{~V}$ to $+383 \mathrm{~V}$ (nine stages at $42.5 \mathrm{~V} /$ stage). Results using the deceleration potential $+315 \mathrm{~V}$ are shown in Fig. 12. The apparatus is designed to use voltages up to $20 \mathrm{kV} / \mathrm{stage}$. A terminal velocity of $2.5 \mathrm{~km} / \mathrm{s}$ would be expected to be reached at $180 \mathrm{kV}$ for the $510 \mathrm{~nm}$ PSLs with $\mathrm{m} / z$ values used in the present experiments. As Hsu and co-workers have shown, [28] it should be straightforward to extend the number of LINAC stages, and the apparatus is already configured to do so.

\section{Impact dynamics and coefficient of restitution for PSL nanoparticles}

In various particle-surface collision models [48-52] of simple collisions (homogenous particles colliding with a flat surface at normal incidence with no rotation) the

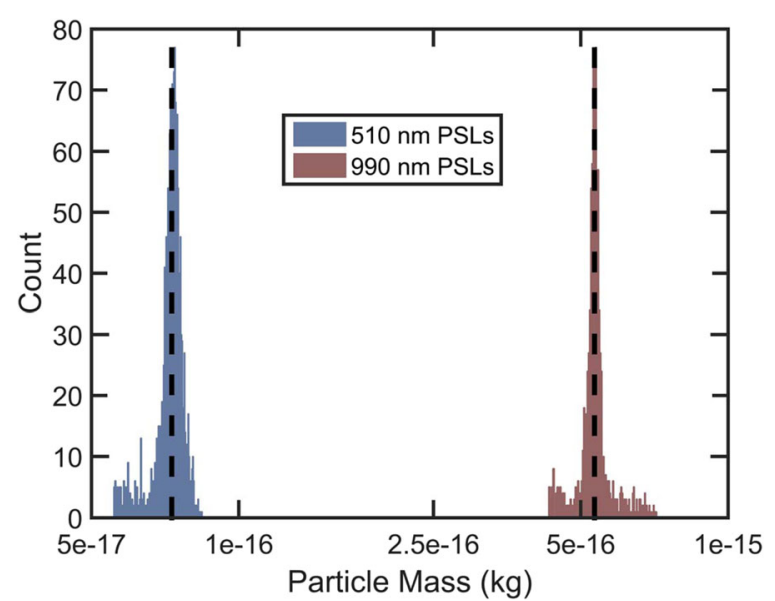

Fig. 10 Mass distribution of $510 \mathrm{~nm}$ PSL (blue) and $990 \mathrm{~nm}$ PSL (red) species, with kinetic energies of $70 \mathrm{eV} /$ $\mathrm{z}$ and $400 \mathrm{eV} / \mathrm{z}$ respectively. Expected mass of each species is shown in black (7.295e-17 kg for $510 \mathrm{~nm}$ PSLs and $5.336 \mathrm{e}-16 \mathrm{~kg}$ for $990 \mathrm{~nm}$ PSLs). For the presented histogram, $510 \mathrm{~nm}$ particles whose mass is more than $\pm 1.3 \sigma$ from the mean are rejected, and $990 \mathrm{~nm}$ particles whose mass is more than $\pm 0.8 \sigma$ from the mean are rejected 


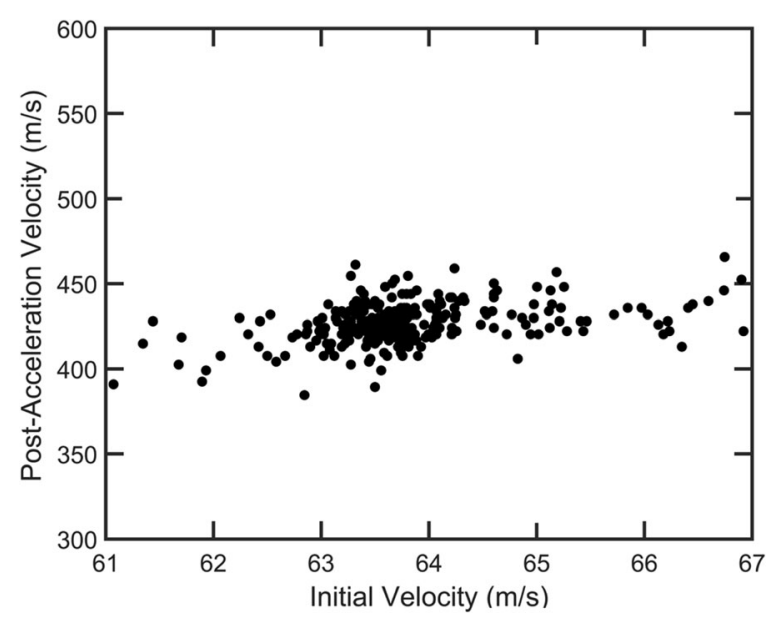

Fig. 11 Accelerated velocity distribution for $990 \mathrm{~nm}$ PSL particles. Initial energy-per-charge of particles is $400 \mathrm{eV}$ with an acceleration potential of $-2500 \mathrm{~V}$ on each of the 9 electrodes

coefficient of restitution, $e$, is an empirical coefficient that fully characterizes the collision. At sufficient distances from the collision surface (to avoid continual particlesurface interaction) $e$ can be defined by:

$$
e^{2}=1-\frac{K E_{\text {incident }}-K E_{\text {rebound }}}{K E_{\text {incident }}}=\left(\frac{v_{\text {rebound }}}{v_{\text {incident }}}\right)^{2}
$$

where $K E$ is the kinetic energy of the particle and $v$ is the particle velocity. This is simply a measure of how the kinetic energy of the particle is partitioned between rebounding kinetic energy and the work of the collision (phonon radiation, target surface waves, plastic deformation of particle, etc.). In general, $e$ can be written as a combination of coefficients associated with each mechanism of irreversible energy loss [50]:

$$
e_{t o t}=1-\sum_{m=1}^{m=n}\left(1-e_{m}^{2}\right)
$$

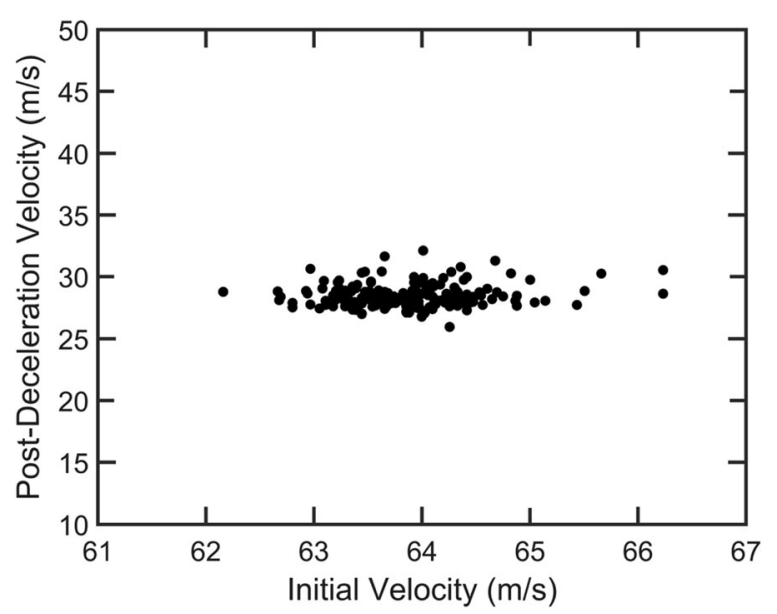

Fig. 12 Decelerated velocity distribution for $990 \mathrm{~nm}$ PSL particles. Initial energy-per-charge of particles is $400 \mathrm{eV}$ with a decelerating potential of $+35 \mathrm{~V}$ on each of the 9 electrodes 


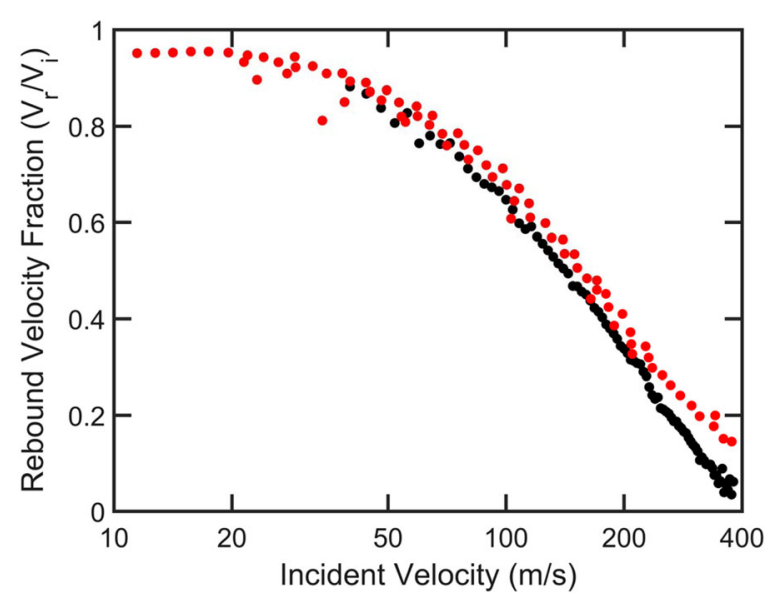

Fig. 13 Coefficient of restitution data for $510 \mathrm{~nm}$ PSLs at normal incidence on silicon, presented with incident velocity grouped in $4 \mathrm{~m} / \mathrm{s}$ bins then averaged (mean), black circles. Any data outside of one standard deviation from the mean for each bin were removed. Created from 4348 measured events. The data are compared to data reported by Dahneke (red circles) in ref. [53] for $1.27 \mu \mathrm{m}$ PSLs at normal incidence on fused silica

where $e_{t o t}$ is described as a combination of an $n$ number of coefficients each associated with energy loss mechanism $m$. Due to the comp lexity in encompassing all possible mechanisms for a given system, no single model exists that accurately predicts a general particle-surface collision. Accordingly, it is valuable to gather coefficient of restitution data for various materials and systems to allow the development of more general models. In Figs. 13 \& 14, the measured coefficients of restitution are presented for $510 \mathrm{~nm}$ and 990 $\mathrm{nm}$ PSLs impacting along the surface normal on a silicon wafer. The data are compared to results presented by Dahneke [50,53] for $1.27 \mu \mathrm{m}$ PSLs on a fused silica substrate. Deviations from the results reported by Dahneke are minor and at this point are likely to be a result of subtle differences in measurement techniques and materials.

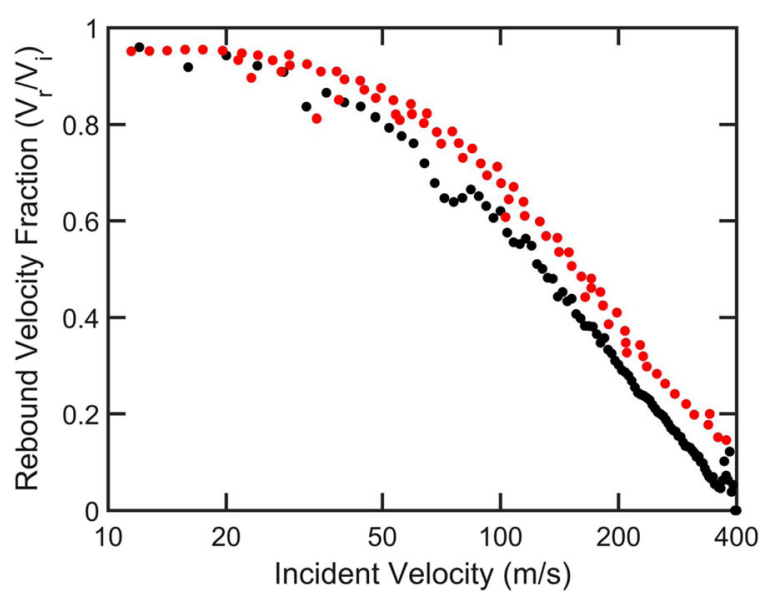

Fig. 14 Coefficient of restitution data for $990 \mathrm{~nm}$ PSLs at normal incidence on silicon, presented with incident velocity grouped in $4 \mathrm{~m} / \mathrm{s}$ bins then averaged (mean), black circles. Any data outside of one standard deviation from the mean for each bin were removed. Created from 3592 measured events. The data are compared to data reported by Dahneke (red circles) in ref. [53] for $1.27 \mu \mathrm{m}$ PSLs at normal incidence on fused silica 


\section{Conclusions}

Formation, trapping, acceleration/deceleration, and coefficient of restitution measurements of highly-charged submicron particles have been demonstrated. Single highly charged PSL spheres have been generated with an ESI source. Subsequent trapping and CDMS analysis of individual particles has been demonstrated. Individual particle acceleration/deceleration has been demonstrated, allowing the acquisition of quantitative coefficient of restitution data. The flexibility of single particle on-the-fly analysis allows the Aerosol Impact Spectrometer to function with a wide range of nanoparticle masses and charges from a given particle source. Additionally, the variable energy selection of the spectrometer allows a variety of particle sources to be implemented in addition to the demonstrated electrospray ionization, including liquid metal ion sources[54-56] and needle-charge dust sources $[57,58]$. The wide range of final energies achievable with the variable linear accelerator/decelerator will allow for a variety of scattering experiments to be performed to examine both hypo- and hypervelocity impact phenomena

\section{Abbreviations}

e: Coefficient of restitution; ESI: Electrospray ionization; FPGA: Field programmable gate array; HV: High voltage;

ICD: Image charge detector; LINAC: Linear accelerator; NET: Nanoparticle electrostatic trap; QD: Quadrupole deflector

\section{Acknowledgements}

This work was supported by the NSF Major Research Instrumentation Instrument Development Program under grant number CHE-1229690 and the Center for Aerosol Impacts on Climate and the Environment (CAICE), a National Science Foundation Center for Chemical Innovation under grant number CHE-1305427. We acknowledge contributions to this project by R. Otto, J.J. Rivera, K.A. Nadler, C.K. Anderson and J. Taulane, as well as discussions with S. De Dea and partial support from Cymer, an ASML Company.

\section{Competing interests}

The authors declare that they have no competing interests.

\section{Authors contributions}

BA and MM participated in the design of the apparatus including the data acquisition system, carried out the measurements and drafted the manuscript. RC conceived of the study, participated in design and coordination and helped draft the manuscript. All authors read and approved the final manuscript.

\section{Publisher's Note}

Springer Nature remains neutral with regard to jurisdictional claims in published maps and institutional affiliations.

\section{Author details}

${ }^{1}$ Department of Nanoengineering, University of California, San Diego, 9500 Gilman Drive, La Jolla, CA 92093-0340, USA. ${ }^{2}$ Department of Chemistry and Biochemistry, University of California, San Diego, 9500 Gilman Drive, La Jolla, CA 92093-0340, USA.

Received: 12 December 2016 Accepted: 9 March 2017

Published online: 11 April 2017

\section{References}

1. Benner WH (1997) A gated electrostatic ion trap to repetitiously measure the charge and $\mathrm{m} / \mathrm{z}$ of large electrospray ions. Anal Chem 69:4162-4168

2. Xie J, Dong M, Li S (2016) Dynamic impact model of plastic deformation between micro-particles and flat surfaces without adhesion. Aerosol Sci Technol 50:321-330

3. Dunn PF, Brach RM, Caylor MJ (1995) Experiments on the low-velocity impact of microspheres with planar surfaces. Aerosol Sci Technol 23:80-95

4. Burchell MJ, Armes SP (2011) Impact ionisation spectra from hypervelocity impacts using aliphatic poly(methyl methacrylate) microparticle projectiles. Rapid Commun Mass Spectrom 25:543-550

5. Mocker A, Bugiel S, Auer S, Baust G, Colette A, Drake K et al (2011) A 2 MV Van de Graaff accelerator as a tool for planetary and impact physics research. Rev Sci Instrum 82:095111

6. Kuuluvainen H, Arffman A, Saukko E, Virtanen A, Keskinen J (2013) A new method for characterizing the bounce and charge transfer properties of nanoparticles. J Aerosol Sci 55:104-115

7. Visser CW, Frommhold PE, Wildeman S, Mettin R, Lohse D, Sun C (2015) Dynamics of high-speed micro-drop impact: numerical simulations and experiments at frame-to-frame times below $100 \mathrm{~ns}$. Soft Matter 11:1708-1722

8. Motoyashiki Y, Hasegawa S, Okudaira K, Sato E (2008) Micrometeoroid impact on ceramic thin components for interplanetary probe. Int J Impact Eng 35:1666-1671 
9. Shelton H, Hendricks CD, Wuerker RF (1960) Electrostatic acceleration of microparticles to hypervelocities. J Appl Phys 31:1243-1246

10. Friichtenicht JF (1962) Two-million-volt electrostatic accelerator for hypervelocity research. Rev Sci Instrum 33:209-212

11. Friichtenicht JF (1964) Micrometeoroid simulation using nuclear accelerator techniques. Nucl Inst Methods 28:70-78

12. Keaton PW, Idzorek GC, Rowton LJ, Seagrave JD, Stradling GL, Bergeson SD et al (1990) A hypervelocity microparticle - impacts laboratory with $100 \mathrm{~km} / \mathrm{s}$ projectiles. Int J Impact Eng 10:295-308

13. Vedder JF (1978) Microparticle accelerator of unique design. Rev Sci Instrum 49:1-7

14. Hendell $E$, Even U (1995) Tabletop linear accelerator for massive molecules. Rev Sci Instrum 66:3901-3902

15. Gamero-Castaño M (2007) Induction charge detector with multiple sensing stages. Rev Sci Instrum 78:043301

16. Fuerstenau SD, Benner WH (1995) Molecular weight determination of megadalton DNA electrospray ions using charge detection time-of-flight mass spectrometry. Rapid Commun Mass Spectrom 9:1528-1538

17. Schultz JC, Hack CA, Benner WH (1998) Mass determination of megadalton-DNA electrospray ions using charge detection mass spectrometry. J Am Soc Mass Spectrom 9:305-313

18. Smith JW, Siegel EE, Maze JT, Jarrold MF (2011) Image charge detection mass spectrometry: Pushing the envelope with sensitivity and accuracy. Anal Chem 83:950-956

19. Doussineau T, Antoine R, Santacreu M, Dugourd P (2012) Pushing the limit of infrared multiphoton dissociation to megadalton-size DNA ions. J Phys Chem Lett 3:2141-2145

20. Contino NC, Pierson EE, Keifer DZ, Jarrold MF (2013) Charge detection mass spectrometry with resolved charge states. J Am Soc Mass Spectrom 24:101-108

21. Keifer DZ, Shinholt DL, Jarrold MF (2015) Charge detection mass spectrometry with almost perfect charge accuracy. Anal Chem 87:10330-10337

22. Doussineau T, Mathevon C, Altamura L, Vendrely C, Dugourd P, Forge V et al (2016) Mass determination of entire amyloid fibrils by using mass spectrometry. Angew Chem Int Ed 55:2340-2344

23. Barney BL, Pratt SN, Austin DE (2016) Survivability of bare, individual Bacillus subtilis spores to high-velocity surface impact: Implications for microbial transfer through space. Planet Space Sci 125:20-26

24. Elliott AG, Merenbloom SI, Chakrabarty S, Williams ER. (2017) Single particle analyzer of mass: A charge detection massspectrometer with a multi-detector electrostatic ion trap. Int J Mass Spec. 414:44-55

25. Keifer DZ, Jarrold MF. Single-molecule mass spectrometry. (2016) Mass Spec Rev. http://dx.doi.org/10.1002/mas. 21495

26. Ring S, Pedersen HB, Heber O, Rappaport ML, Witte PD, Bhushan KG et al (2000) Fourier transform time-of-flight mass spectrometry in an electrostatic ion beam trap. Anal Chem 72:4041-4046

27. Johnson CJ, Shen BB, Poad BLJ, Continetti RE (2011) Photoelectron-photofragment coincidence spectroscopy in a cryogenically cooled electrostatic ion beam trap. Rev Sci Instrum 82:105105

28. Hsu YF, Lin JL, Lai SH, Chu ML, Wang YS, Chen CH (2012) Macromolecular lon Accelerator. Anal Chem 84:5765-5769

29. Schoner C, Rennecke S, Weber AP, Poschl T (2014) Introduction of a new technique to measure the coefficient of restitution for nanoparticles. Chem Ing Tech 86:365-374

30. Eurov DA, Kurdyukov DA, Stovpiaga EY, Salasyuk AS, Jager J, Scherbakov AV et al (2014) Hypersonic properties of monodisperse spherical mesoporous silica particles. J Phys D Appl Phys 47:335303

31. Zajfman D, Heber O, Vejby-Christensen L, Ben-Itzhak I, Rappaport M, Fishman R et al (1997) Electrostatic bottle for long-time storage of fast ion beams. Phys Rev A 55:R1577-R1580

32. Dahan M, Fishman R, Heber O, Rappaport M, Altstein N, Zajfman D et al (1998) A new type of electrostatic ion trap for storage of fast ion beams. Rev Sci Instrum 69:76-83

33. Bogan MJ, Benner WH, Hau-Riege SP, Chapman HN, Frank M (2007) Aerosol sample preparation methods for $\mathrm{X}$-ray diffractive imaging: Size-selected spherical nanoparticles on silicon nitride foils. Aerosol Sci 38:1119-1128

34. Benner WH, Bogan MJ, Rohner U, Boutet S, Woods B, Frank M (2008) Non-destructive characterization and alignment of aerodynamically focused particle beams using single particle detection. Aerosol Sci 39:917-928

35. Kerby JD, Terik Daly R, Austin DE (2013) A novel particle source based on electrospray charging for dust accelerators and its significance for cosmic dust studies. Earth Planets Space 65:157-165

36. Daly RT, Kerby JD, Austin DE (2013) Electrospray charging of minerals and ices for hypervelocity impact research. Planet Space Sci 75:182-187

37. Liu P, Ziemann PJ, Kittelson DB (1995) Generating particle beams of controlled dimensions and divergence: I. Theory of particle motion in aerodynamic lenses and nozzle expansions. Aerosp Sci Technol 22:293-313

38. Liu P, Ziemann PJ, Kittelson DB (1995) Generating particle beams of controlled dimensions and divergence: II. Experimental evaluation of particle motion in aerodynamic lenses and nozzle expansions. Aerosp Sci Technol 22:314-324

39. Wang X, McMurry PH (2006) A design tool for aerodynamic lens systems. Aerosp Sci Technol 40:320-334

40. Dahl DA (2000) SIMION for the personal computer in reflection. Int J Mass Spectrom 200:3-25

41. Cai Y, Peng WP, Kuo SJ, Lee YT, Chang HC (2002) Single-Particle Mass Spectrometry of Polystyrene Microspheres and Diamond Nanocrystals. Anal Chem 74:232-238

42. Doussineau T, Santacreu M, Antoine R, Dugourd P, Zhang W, Chaduc I et al (2013) The charging of micellar nanoparticles in electrospray ionization. ChemPhysChem 14:603-609

43. Ouadah N, Doussineau T, Hamada T, Dugourd P, Bordes C, Antoine R (2013) Correlation between the charge of polymer particles in solution and in the gas phase investigated by zeta-potential measurements and electrospray ionization mass spectrometry. Langmuir 29:14074-14081

44. Rayleigh LXX (1882) On the equilibrium of liquid conducting masses charged with electricity. Philos Mag Ser 5 14:184-186

45. Fenn JB, Mann M, Kai Meng C, Fu Wong S, Whitehouse CM (1989) Electrospray ionization for mass spectrometry of large biomolecules. Sci New Ser 246:64-71

46. Vazquez G, Alvarez E, Navaza JM (1995) Surface tension of alcohol water + water from 20-50 degrees C. J Chem Eng Data 40:611-614 
47. Doussineau T, Desert A, Lambert O, Taveau JC, Lansalot M, Dugourd P et al (2015) Charge detection mass spectrometry for the characterization of mass and surface area of composite nanoparticles. J Phys Chem C 119:10844-10849

48. Wall S, John W, Wang H-C, Goren SL (1990) Measurements of kinetic energy loss for particles impacting surfaces. Aerosol Sci Technol 12:926-946

49. Tsai C-J, Pui DYH, Liu BYH (1990) Capture and rebound of small particles upon impact with solid surfaces. Aerosol Sci Technol 12:497-507

50. Dahneke B (1995) Particle bounce or capture - search for an adequate theory: I. Conservation-energy-model for a simple collision process. Aerosol Sci Technol 23:25-39

51. Andres RP (1995) Inelastic energy transfer in particle/surface collisions. Aerosol Sci Technol 23:40-50

52. Valentini P, Dumitrica T (2007) Microscopic theory for nanoparticle-surface collisions in crystalline silicon. Phys Rev B 75:224106

53. Dahneke B (1975) Further measurements of the bouncing of small latex spheres. J Colloid Interface Sci 51:58-65

54. Vladimirov W, Badan VE, Gorshkov VN, Soloshenko IA (1993) Liquid metal microdroplet source for deposition purposes. Appl Surf Sci 65/66:1-12

55. Bischoff I, Akhmadaliev C, Mair AWR, Mair GLR, Ganetsos T, Aidinis CJ (2004) Investigation of a tin liquid metal ion source. Appl Phys A 79:89-92

56. Jaworek A (2007) Micro- and nanoparticle production by electrospraying. Powder Technol 176:18-35

57. Stubig M, Schafer G, Ho T-M, Srama R, Grun E (2001) Laboratory simulation improvements for hypervelocity micrometeorite impacts with a new dust particle source. Planet Space Sci 49:853-858

58. Shu A, Collette A, Drake K, Gruen E, Horanyi M, Kempf S et al (2012) 3 MV hypervelocity dust accelerator at the Colorado Center for Lunar Dust and Atmospheric Studies. Rev Sci Instrum 83:075108

\section{Submit your manuscript to a SpringerOpen ${ }^{\circ}$ journal and benefit from:}

- Convenient online submission

Rigorous peer review

- Immediate publication on acceptance

- Open access: articles freely available online

- High visibility within the field

Retaining the copyright to your article

Submit your next manuscript at $>$ springeropen.com 